\title{
Bayesian count regression analysis for determinants of antenatal care service visits among pregnant women in Amhara regional state, Ethiopia
}

\author{
Mekuanint Simeneh Workie ${ }^{1 *}$ and Ayenew Molla Lakew ${ }^{2}$
}

*Correspondence:

mekuanintsimeneh@gmail. com

${ }^{1}$ Mathematical and Statistical Modeling, Debre Markos University, Debre Markos, Ethiopia

Full list of author information is available at the end of the article

\begin{abstract}
Background: Complications of pregnancy and childbirth are a leading cause of maternal morbidities and mortalities in developing countries. World Health Organization (WHO) estimates that over 500,000 women and girls die each year from the complications. Despite proven interventions that could prevent death or disability during pregnancy and childbirth, maternal mortality remains a major burden in many developing countries, including Ethiopia. This study aimed to assess the status of antenatal care utilization and modeling Bayesian Count Regression model for the determinants of utilization of antenatal care services visits among pregnant women in Amhara regional state.
\end{abstract}

Methods: It was a community based analytical cross-sectional study, conducted in Amhara region among women in the reproductive age group (age 15-49). The analysis was based on data from women who had at least one birth during the 5 years preceding the survey. The source of data was the 2014 Ethiopia Demographic and Health Survey which was accessed from Central Statistical Agency. Bayesian analytic approach was applied to model the mixture data structure inherent in zero-inflated count data by using the zero-inflated Poisson model.

Results: About $37 \%(95 \% \mathrm{Cl} 0.32,0.42)$ of the pregnant mothers were not received antenatal care services during their pregnancy and about $23 \%$ of them were visited at least four times. From Bayesian zero inflated Poisson regression it was found that rural pregnant women $(\mathrm{OR}=1.13$; HPD Cl 1.12, 1.44), women who can read and write

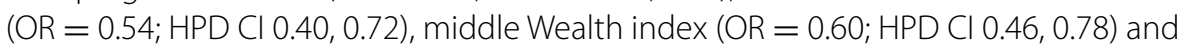
media exposures (OR $=0.72 ; \mathrm{HPD}: 0.56,0.92)$ were statistically associated with no ANC visits.

Conclusions: About three-fourth pregnant mothers were not receive adequate number of visits recommended by the World Health Organization. Mother's education, media exposure, residence and wealth index were significant predictors of ANC service utilization. This research suggests that to reduce the inadequate number of ANC visits in Amhara region, attention should be given to women with low educational status and rural women. 
Keywords: Bayesian approach, Classical approach, ANC, Amhara region, MCMC, Posterior distribution, Prior density

\section{Background}

Antenatal care (ANC) for pregnant women by health professionals maintains women's health during pregnancy and improves pregnancy outcomes by identifying and managing pregnancy related complications [1-3]. ANC services offer pregnant women an entry point to the health care system, providing appropriate screening, intervention and treatment throughout pregnancy, and encouraging women to seek a skilled birth attendant for their delivery [4]. Women attending ANC visits receive sufficient evidencebased clinical interventions [5]. This is useful in optimizing maternal and fetal outcome through regular monitoring of pregnancy [6].

The World Health Organization (WHO) recommends all women with uncomplicated pregnancies to attend four ANC visits during the course of the pregnancy to prevent and identify pregnancy risks and treat conditions timely through providing appropriate information to the client. During their visit they should receive tetanus toxoid immunization, intermittent preventive treatment of malaria, deworming, iron and folic acid, and insecticide treated bednet [7].

Pregnant women can also be screened for signs associated with high probability of complications and subsequent specialized care can be arranged. The WHO estimates that over 500,000 women and girls die each year from the complications. These high morbidity and mortality rates make maternal health a huge public health problem in the developing countries. Developing countries shares $99 \%$ of the burden in which around $56 \%$ of these maternal deaths took place in Sub-Saharan Africa. According to the EDHS 2011, the maternal mortality ratio in Ethiopia was estimated to be $676 / 100,000$ live births, which is much higher than many Sub-Sahara African countries [8-10].

Currently, $71 \%$ of women worldwide receive any ANC, in industrialized countries more than $95 \%$ of pregnant women have access to ANC. In sub-Saharan Africa, $69 \%$ of pregnant women have at least one ANC visit [11]. While different studies have looked at diverse risk factors for antenatal care (ANC) and delivery service utilization in the country, Ministry of health of Ethiopia in 2007 reported that about 52\% Ethiopian women received one or more ANC visits, less than $17 \%$ received professionally assisted delivery care and 19\% received postnatal care [12]. The major causes for poor health in developing countries are inadequate access and under-use of modern health care services. The number of antenatal care visits during the last pregnancy by mothers aged 15-49 years was analyzed. More than $55 \%$ of the mothers did not use antenatal care (ANC) services, while more than $22 \%$ of the women used antenatal care services less than four times. More than half of the women (52\%) who had access to health services had at least four antenatal care visits. The zero-inflated negative binomial model was found to be more appropriate for analyzing the data [13].

According to the 2014 mini EDHS report 32 percent of women with a live birth in the five years before the survey were received four or more ANC visits which is a marked improvement from 19\% reported in the 2011 EDHS [10, 14]. A study in Rwanda and slum residents of Addis Ababa explained that about 46-81.6\% of women were received 
at least four ANC visits respectively [15, 16]. Only $43.1 \%$ of women in Amhara region received ANC from skilled provider [14].

Addressing this situation requires a substantial improvement in the coverage and quality of maternal health care services in the country. In Ethiopia although access to health care services is improving, the country has faced challenges in increasing health care utilization [17]. It is thus important to examine the extent to which women are making use of the services and answer why many women do not use recommended services.

Despite the fact that antenatal care seeking is essential, little is known about status and factors affecting level of ANC visit in Amhara region. Thus, detail understanding on the factors affecting in the number of ANC service utilization is crucial. EDHS 2011, Information coming from community-based studies related to the Health Extension Programme (HEP) in rural areas is still very limited due to different factors. That is why this study is aimed to statistically analyze the determinants of the barriers in antenatal care service visits among pregnant women in Amhara region. Furthermore, this study will provide valuable information about count data models.

\section{Methodology}

It was a community based analytical cross-sectional study. The data were collected from a woman in the reproductive age group (age 15-49) from all zones in Amhara region. The study subjects are women aged 15-49 years who gave at least one birth in the last 5 years preceding the survey. The data used for this study was taken from the 2014 EMDHS which is a nationally representative survey of women in the fertile age (15-49 years age) groups taken from the CSA. It is the fourth comprehensive survey and designed to provide estimates for the health and demographic variables of interest for the following domains: Amhara region as a whole, urban and rural area of the region. The objective of 2014 Ethiopia Mini Demographic and Health Survey was to collect population-based data on key demographic indicators to support the monitoring and evaluation needs for Phase IV of the health sector development program (HSDP).

\section{Variables of the study}

The dependent variable of this study is the number of ANC service visits of pregnant women from early pregnancy to their 9 months of pregnancy period. This research attempts to include socioeconomic, demographic, health and environmental related factors that are assumed as a potential determinant for the barriers in the number of antenatal care service visits. These factors are presented: Wealth index, Utilization of media, Type of residence, Age of mother's, Mother's education, marital status, Birth order and sign of pregnancy complications.

\section{Statistical models}

Analysis of count data can be done in various fields, including health science. Several models have been developed in recent decades to analyze these count data, especially ANC service visits of pregnant women data. Modeling these data begins with the Poisson regression model has been used for several years. However, because the Poisson distribution has the same mean and variance, it often does not reflect typical characteristics such as substantial over-dispersion contained in the count data. A great deal of 
work regarding model building for ANC service visits of pregnant women data has been based on the negative binomial regression model. We often encounter situations where these count data have a tendency to contain a large portion of zero observations. We call these data zero-inflated data, if the number of zeros is greater than expected when using the standard PRM or NBRM [18]. As a result, the zero-inflated Poisson (ZIP) and zero inflated negative binomial (ZINB) regression models have received considerable attention when used in ANC service visits pregnant women's studies. These models are motivated by the fact that traditional applications of PRM and NBRM do not address the possibility of zero inflated counting processes. Zero inflation can be explained by the existence of a dual-state process for ANC data generation. There are situations where a major source of over dispersion is a preponderance of zero counts, and the resulting over dispersion cannot be modeled accurately with the negative binomial model. In such scenarios, one can use zero-inflated Poisson or zero-inflated negative binomial model to fit the data [19].

\section{Zero-inflated Poisson regression models}

ZIP model operates on the principle that the excess zero density that cannot be accommodated by a traditional countable structure. The probability of an ANC visitation entity being in zero or non-zero states can be accounted by a splitting regime that models a woman who is not visited for ANC versus a woman who has visited for the ANC during their pregnancy period determined by a binary logit model $[20,21]$. In one regime $\left(R_{1}\right)$ the outcome is always a zero count, while in the other regime $\left(R_{2}\right)$ the counts follow a standard Poisson process.

Suppose that: $\mathrm{p}\left(\mathrm{y}_{\mathrm{i}} \in \mathbf{R}_{\mathbf{1}}\right)=\mathrm{p}_{\mathrm{i}} ; \mathrm{p}\left(\mathrm{y}_{\mathrm{i}} \in \mathbf{R}_{\mathbf{2}}\right)=1-\mathrm{p}_{\mathrm{i}} ; \mathrm{i}=1,2,3, \ldots, \mathrm{n}$ where $\mathrm{p}_{\mathrm{i}}=$ inflation probability. This two-state process gives a simple two-component mixture distribution with PMF:

$$
\mathrm{p}\left(\mathrm{Y}_{\mathrm{i}}=\mathrm{y}_{\mathrm{i}}\right)= \begin{cases}p_{i}+\left(1-p_{i}\right) e^{-\lambda_{i}}, & \text { if } y_{i}=0 \\ \left(1-p_{i}\right) \frac{e^{-\lambda_{i}} \lambda_{i}^{y_{i}}}{y_{i} !} & \text { if } y_{i}>0\end{cases}
$$

The mean and variance of the ZIP random variable are $\mathrm{E}\left(\mathrm{y}_{\mathrm{i}}\right)=\left(1-\mathrm{p}_{\mathrm{i}}\right) \lambda_{\mathrm{i}}$ and $\operatorname{var}\left(y_{i}\right)=\left(1-p_{i}\right) \lambda_{i}\left(1+p_{i} \lambda_{i}\right)$. This indicates that the distribution of $y_{i}$ exhibits over dispersion when $p_{i}>0$. It is clear that this reduces to the standard Poisson distribution when $\mathrm{p}_{\mathrm{i}}=0$. Positive values of $\mathrm{p}_{\mathrm{i}}$ imply that there is a zero-inflation. Analogously, the pmf of the ZINB is the result of a Bernoulli trial is used to determine which of the two processes are used. For mother, $\mathrm{I}$, with probability $\mathrm{P}_{\mathrm{i}}$ the only possible response of the first process is zero counts, and with the probability of $\left(1-\mathrm{P}_{\mathrm{i}}\right)$ the response of the second process is governed by a negative binomial with mean $\lambda_{\mathrm{i}}$. The overall probability of zero counts is the combined probability of zeros from the two processes. For zeroinflated and over dispersed data a frequent modeling choice is the Zero-Inflated Negative Binomial (ZINB) model. The response variable $Y_{i}=(1,2 \ldots n)$ has a pmf given. A ZINB model for the response (the number of ANC visits during pregnancy) can be written as: 


$$
\mathrm{p}(\mathrm{Yi}=\mathrm{yi})= \begin{cases}p_{i}+\left(1-p_{i}\right)\left(1+\gamma \lambda_{i}\right)^{\frac{1}{k}} & \text { if } y_{i}=0 \\ \left(1-p_{i}\right) \frac{\Gamma\left(y_{i}+\frac{1}{k}\right)}{\Gamma\left(y_{i}+1\right)} \frac{\left(1+\gamma \lambda_{i}\right)^{y_{\mathrm{i}}}}{\left(k \lambda_{i}+1\right)^{\left(y_{i}+\frac{1}{\mathrm{k}}\right)}} & \text { if } y_{i}>0\end{cases}
$$

where $0 \leq \mathrm{p}_{\mathrm{i}} \leq 1$ and $\lambda_{\mathrm{i}}>0$, is the dispersion parameter with $\gamma>0$ and $\Gamma($.$) is the gamma$ function. The mean and the variance of the model are defined as $E\left(y_{i}\right)=\left(1-p_{i}\right) \lambda_{i}$ and $\operatorname{var}\left(y_{\mathrm{i}}\right)=\left(1-\mathrm{p}_{\mathrm{i}}\right) \lambda_{\mathrm{i}}\left(1+\lambda_{\mathrm{i}}\left(\mathrm{p}_{\mathrm{i}}+\mathrm{k}\right)\right)$ The ZINB distribution reduces to the ZIP distribution as $\mathrm{k} \rightarrow 0$. The parameter $\lambda_{\mathrm{i}}$ is modeled as a function of a linear predictor, that is, $\lambda_{i}=\exp \left(X_{i}^{\mathrm{T}} \beta\right)$. For common choice and simplicity, $\mathrm{p}_{\mathrm{i}}$ is characterized in terms of a logistic regression model by writing as logit $(\mathrm{pi})=\mathrm{X}_{\mathrm{i}}^{\mathrm{T}} \alpha . \alpha$ is the $(\mathrm{q}+1) \times 1$ vector of of zeroinflated coefficients to be estimated, associated with the known zero-inflation covariate vector $\mathrm{X}_{\mathrm{i}}^{\mathrm{T}}$, where $\mathrm{q}$ is the number of the covariates X's not including the intercept. In the terminology of generalized linear models (GLMs), $\log \left(\lambda_{\mathrm{i}}\right)$ and $\operatorname{logit}\left(\mathrm{p}_{\mathrm{i}}\right)$ are the natural links for the negative binomial mean and Bernoulli probability of success [21].

The standard estimation technique for the ZIP and the ZINB is based on the maximum likelihood estimation. The maximum likelihood estimation method is used to assess the adequacy of any two or more than two nested models by using the likelihood ratio test. As a result this test of statistics will be compared with the tabulated Chi square with a degree of freedom, the difference between the degree of freedom of the model under the null hypothesis and the alternative hypothesis respectively. This method is not appropriate for models which are not nested one on the other, in such situation; we will use another method such as the Akaike information criteria (AIC) and Bayesian information criteria [22]. After the fitting Poisson regression, Negative Binomial regression, ZIP and ZINP regression to the data, we can ask the question: what is the best model for our analysis? As a response, many tests are developed. In this we will focus on the Vuong test. The Vuong test is a non-nested test that is based on a comparison of the predicted probabilities of two models that do not nest [23]. For instance, comparisons between Zero inflated count models with ordinary Poisson, or Zero inflated negative binomial against ordinary negative binomial model can be done using Voung test.

\section{Bayesian inference}

A Bayesian approach is a useful tool in statistics, where all forms of uncertainty are expressed in terms of probability. The idea of Bayesian statistics within the context of life data analysis is to integrate prior knowledge, along with a given set of current observations, in order to make statistical inferences. The advantages of Bayesian inference are well known and include elicitation of prior beliefs about past experience, avoidance of asymptotic approximations and practical estimation of functions of parameters [24, 25]. Bayesian inference assumes that the data are fixed and consider all unknown parameters as random variables [26]. Studied zero-inflated distributions with the Bayesian point of view using the data augmentation algorithm [27], developed a full Bayesian estimation method of zeroinflated regression model by Gibbs sampling. Analyze zero-inflated models with a Bayesian approach to estimate regression parameters and compare prediction performances with the frequentist approach for applications to ANC visits counter measures. A popular way of simulating from a general posterior distribution is by using $\mathrm{MCMC}$ methods. 


\section{Bayesian ZIP regression models}

Bayesian approach may be applied to a zero-inflated Poisson regression model taking account of the uncertainty in the parameters. This approach considers prior information about the distribution of parameters along with the likelihood of the observed data to construct a posterior distribution of relevant quantities for inference about unknown parameters. Regression-type models are widely used in applied research to adjust for covariate effects and assess relationships between key predictors and the response. While conventional regression models contain only one set of predictors for inference about a single response, covariates typically enter a ZIP regression model at both the Bernoulli zero-inflation and Poisson count stages, yielding two sets of parameters corresponding to $\mathrm{p}$ and $\lambda$. Thus, this allows simultaneous inferences to be made about the zero-inflation and count process. In the usual specification, covariates are related to $\lambda$ through a loglinear model, and to $\mathrm{p}$ through a logit model. Specifically,

$$
\begin{aligned}
& \operatorname{logit}(\mathrm{p})=\alpha_{0}+\alpha_{1} x_{12}+\cdots+\alpha_{\mathrm{m}} \mathrm{x}_{1 \mathrm{~m}} \\
& \log (\lambda)=\beta_{0}+\beta_{1} \mathrm{x}_{21}+\cdots+\beta_{\mathrm{k}} \mathrm{x}_{2 \mathrm{k}}
\end{aligned}
$$

where $\mathrm{X}_{1}=\left(1, \mathrm{X}_{11}, \mathrm{X}_{12}, \ldots, \mathrm{X}_{1 \mathrm{~m}}\right)$ is the covariate vector included in the zero stage and (1, $\left.X_{21}, X_{22}, \ldots, X_{2 k}\right)$ is the covariate vector included in the Poisson stage. $\alpha=\alpha_{0}, \alpha_{1}, \ldots, \alpha_{m}$ and $\beta=\beta_{0}, \beta_{1}, \ldots, \beta_{\mathrm{k}}$ are the corresponding coefficient vectors. The observed data are assumed to be independent. For other situations, where the independence assumption is relaxed, a correlation structure can be modeled by introducing random terms [28]. With covariates included in the ZIP regression model, the likelihood function for a random sample $\mathrm{Y}=\left(\mathrm{y}_{1}, \mathrm{y}_{2}, \ldots, \mathrm{y}_{\mathrm{n}}\right) \sim \operatorname{ZIP}\left(\lambda_{\mathrm{j}}=\exp \left(\mathrm{x}_{2 \mathrm{j}} \beta\right), \mathrm{p}_{\mathrm{j}}=\frac{\exp \left(x_{1 j} \alpha\right)}{\exp \left(1+x_{1 j} \alpha\right)}\right.$ is re parametrized as

$$
\mathrm{f}(\mathrm{y} / \alpha, \beta)=\left[\prod_{j=1}^{L}\left(p_{i}+\left(1-p_{i}\right) e^{-\lambda_{i}}\right)\right] \times\left[\prod_{j=l+1}^{n}\left(1-p_{i}\right) \frac{e^{-\lambda_{i}} \lambda_{i}^{y_{i}}}{y_{i} !}\right]
$$

where $\mathrm{n}$ represents the total number of observations for $\mathrm{Y}$ among which a subset of the first respondents have zero values. The $\mathrm{y}_{\mathrm{L}+1}, \ldots, \mathrm{y}_{\mathrm{n}}$ indicate observations with non zero values With covariates added, the prior is assigned on the regression coefficients for the log-linear model (i.e., the Poisson count stage) and the logistic model (i.e., the Bernoulli zero-inflation stage) as outlined earlier. Without prior knowledge about the distribution of the parameter, Bayesian may choose non-informative priors. In doing so, the role of the prior distribution is minimized and more weight is given to the data in determining the posterior distribution. In the case of no available prior knowledge, we consider a normal distribution with mean $\mu=0$ and large variance. In this essay, we choose $\theta^{2}=1000$. For example, with an independent normal $\left(\mu, \theta^{2}\right)$ prior for each element in $\alpha$ and $\beta$ with a large value of $\theta$ to reflect the lack (or ignorance) of prior information, we have the following joint prior distribution:

$$
f(\alpha, \beta)=\prod_{j=1}^{m}\left[\frac{1}{\sqrt{2 \pi \sigma_{\alpha j}^{2}}} \exp \left\{\frac{-1}{2}\left(\frac{\alpha_{j}-\mu_{\alpha j}}{\sigma_{\alpha j}}\right)^{2}\right\}\right] \times \prod_{j=0}^{k} \frac{1}{\sqrt{2 \pi \sigma_{\beta j}^{2}}} \exp \left\{\frac{-1}{2}\left(\frac{\beta_{j}-\mu_{\beta j}}{\sigma_{\beta j}}\right)^{2}\right\} .
$$


In Bayesian approach, interest lies in estimating posterior distributions of model parameters rather than individual parameter values and asymptotic standard errors. Applying Bayes rule to the ZIP regression model with co-varieties based on the likelihood function in Eq. (5) and the prior distribution function in Equation (6) yield the following joint posterior distribution with a nonstandard density:

$$
f(\alpha, \beta / y) \propto f(y / \alpha, \beta) f(\alpha, \beta) .
$$

This was a complex posterior distribution that is complicated to converge to a known distribution.

\section{The Gibbs sampling algorithm}

The Gibbs sampler introduced by [25] is a special case of Metropolis-Hasting algorithm where the random value is always accepted (i.e. $a=1$ ). Using as proposal density result in acceptance probability parameter $=1$, and therefore the proposed move is accepted in all iterations. One advantage of the Gibbs sampler is that, at each step, random values must be generated from uni-variate distribution for which a wide variety of computational tools exist [29]. Frequently, these conditional distributions have a known form and, thus, random numbers can be easily simulated using standard functions in statistics and computing software. Gibbs sampling is always moving to new values and, most importantly, does not require specification of proposal distributions. On the other hand, it can be ineffective when the parameter space is complicated or the parameters are highly correlated. Because of the difficulty in analytically determining the posterior distribution, simulation techniques are generally used to obtain posterior information. Suppose that $b$ and $a$ are a vector of $\mathrm{k}$ and $\mathrm{m}$ parameters respectively. Moreover, suppose that we can simulate random variates from each conditional density. In the case of the ZIP regression model with Co-varying [30], the Gibbs sampler can proceed with the following ways:

Algorithm 1: Gibbs Sampling Algorithm: ZIP model with covariates

1. Set initial value $: \alpha_{0}{ }^{(0)}, \alpha_{1}{ }^{(0)}, \ldots, \alpha_{\mathrm{m}}{ }^{(0)}$ and $\beta_{0}{ }^{(0)}, \ldots, \beta_{\mathrm{k}}{ }^{(0)}$

2. Sample from the full conditional distribution according to $\mathrm{i}=0,1,2, \ldots, \mathrm{n}-1$

$$
\begin{gathered}
\text { Simulate: } \alpha_{0}{ }^{(\mathrm{i}+1)} \sim \pi_{0}\left(\alpha_{0}^{i} / \alpha_{1}^{i}, \ldots, \alpha_{m}^{i}{ }^{\prime} \beta_{0}{ }^{(\mathrm{i})}, \cdots, \beta_{\mathrm{k}}{ }^{(\mathrm{i})}, \mathrm{Y}, \mathrm{X}\right) \\
\text { - } \\
\text { - } \\
\text { Simulate: } \beta_{\mathrm{k}}{ }^{(\mathrm{i}+1)} \sim \pi_{k}\left(\beta_{\mathrm{k}}^{(\mathrm{i}+1)} / \alpha_{0}^{(i+1), \ldots,} \beta_{\mathrm{k}-1}{ }^{(\mathrm{i}+1)}, \mathrm{Y}, \mathrm{X}\right)
\end{gathered}
$$

3. Return to step 2 until convergence

The Bayesian inference using Gibbs sampling (BUGS) project has developed a flexible software for Bayesian analysis of complex statistical models by using MCMC methods [30]. We use these tools to estimate the ZIP regression models. Furthermore, we may compute other statistics of the posterior such as, median or quantile, credible 
interval, mode etc. In addition from this sample, we can compute and observe correlations between parameters, produce desired plot of the marginal posterior distributions, etc.

\section{Convergence of the algorithm}

Flexible software for Bayesian analysis of complex statistical models by using MCMC methods. We use these tools to estimate the ZIP regression models. The term convergence of an MCMC algorithm refers to whether the algorithm has reached its equilibrium (target) distribution. Hence, monitoring the convergence of the algorithm is essential for producing results from the posterior distribution of interest. Convergence diagnosis was adopted to answer the equation of how to determine whether the sampler has reached its stationary distribution [31]. Generally it is unclear how much we must run an algorithm to obtain samples from the correct target distributions. Several diagnostic tests have been developed to monitor the convergence of the algorithm. The simplest way is to monitor the MC error since small values of this error will indicate that we have calculated the quantity of interest with precision. Monitoring auto correlation is also very useful since low or high values indicate fast or low convergence, respectively. A second way is to monitor the trace plots: the plots of the iterations versus the generated values. If all values are within a zone without strong periodicity and (specially) tendencies, then we can assume convergence. A poor choice of starting values and/ or proposal distribution can greatly increase the required burn-in time. To show evidence for poor mixing, time series trace was seen for a minimum burn-in period for some starting value. Various convergence tests will be used to assess whether stationary has indeed been reached [32]. The Gelman-Rubin convergence diagnostic was multiple chains generated simultaneously, the diagnostic test is applied by computing and comparing within-sample variability and between-sample variability.

\section{Results}

The descriptive statistics given in the Table 1 shows the number and percentage of ANC visits that the pregnant mothers in the sample have encountered in their 9 months of pregnancy period. From this study $136(37.00 \%)$ of the pregnant mothers had not been

Table 1 Number of mothers that experienced ANC visits in Amhara National Regional State, 2014

\begin{tabular}{lccc}
\hline No_ANC visits & Count & Percent & $\begin{array}{c}\text { Cumulative } \\
\text { percent }\end{array}$ \\
\hline 0 & 136 & 37.0 & 37.00 \\
1 & 43 & 11.7 & 48.60 \\
2 & 35 & 9.5 & 58.20 \\
3 & 71 & 19.5 & 77.40 \\
4 & 40 & 10.9 & 88.30 \\
5 & 18 & 4.9 & 93.20 \\
6 & 14 & 3.8 & 97.00 \\
7 & 7 & 1.9 & 98.90 \\
8 & 2 & 0.5 & 99.50 \\
9 & 1 & 0.3 & 99.70 \\
10 & 1 & 0.3 & 100.00 \\
\hline
\end{tabular}


received ANC during their period of pregnancy, where as 43 (11.7\%) of them visited only once, 35 (9.5\%) of them visited twice, 71 (19.50\%) visited three times, 40 (10.90\%) visited four times and etc.

This indicates an over dispersion of the response variable. Since the histogram is highly peaked at zero, we can state that the over dispersion is due to excess of zeroes (Fig. 1). Due to a large number of zero outcomes, the histogram is highly picked at the very beginning (about the zero values). This leads to have a positively (or right) skewed distribution. This was an indication that the data could be fitted better by count data models which takes into account excess zeroes.

\section{Test for over dispersion}

In Poisson regression analysis, deviance and Pearson Chi square goodness of fit statistics indicate there was over dispersion (Table 2). Since the Pearson Chi square statistic divided by the degrees-of-freedom is higher than one and the observed value of 2.63, then the mentioned goodness of statistics represents that there was an over dispersion in the data set.

Even if the Deviance and Pearson Chi square goodness of fit statistics of 333.07 and 324.96 respectively in Negative Binomial regression is dropped considerably, still

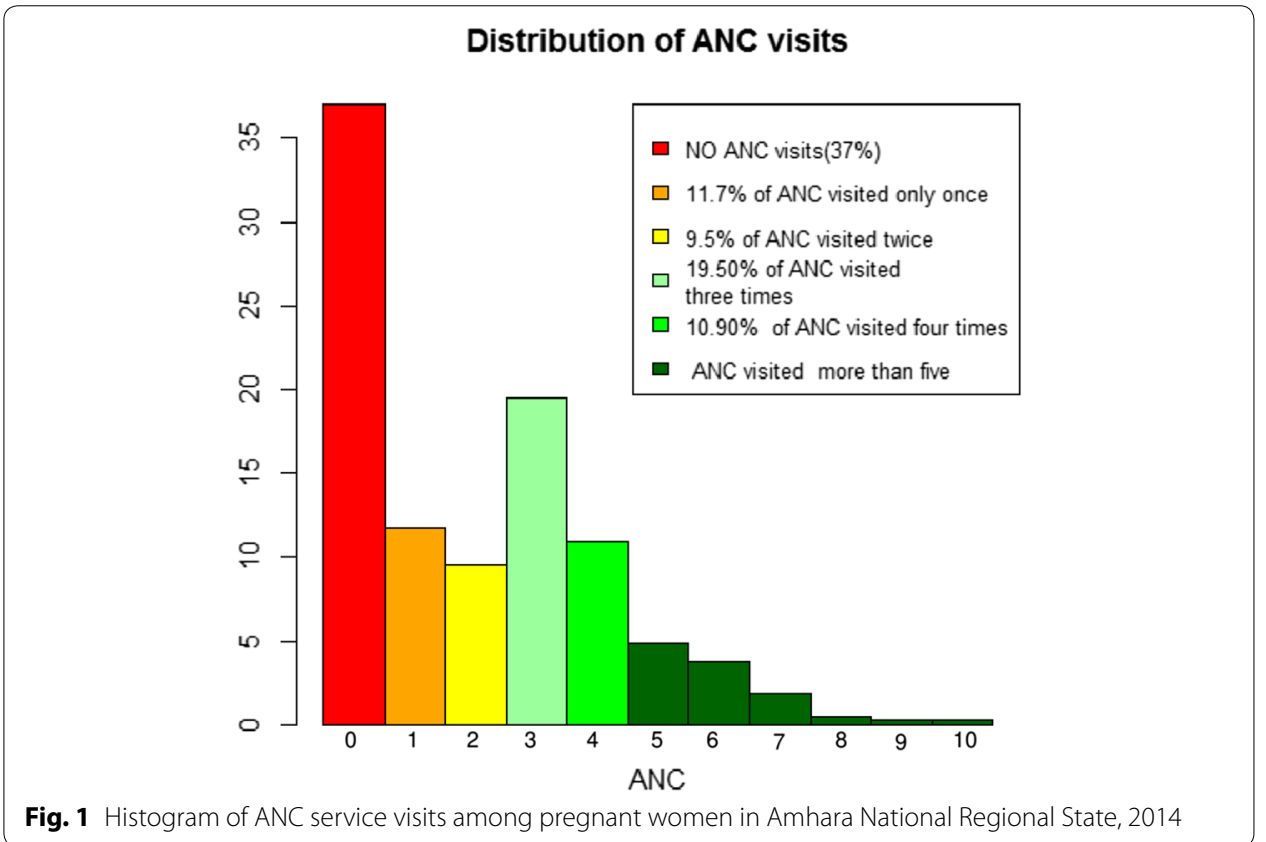

Table 2 Test for Over dispersion

\begin{tabular}{llll}
\hline Models & Df & Value & Value/Df \\
\hline Deviance & & & \\
Poisson & 362 & 845.7467 & 2.3363 \\
NegBin & 362 & 333.0707 & 0.9201 \\
Pearson & & & \\
Poisson & 362 & 950.9179 & 2.6268 \\
NegBin & 362 & 324.9572 & 0.8977 \\
\hline
\end{tabular}


Table 3 Test for the comparison of the models using LRT and Voung test

\begin{tabular}{|c|c|c|c|c|}
\hline$M_{1}$ & $M_{2}$ & V-statistic (p-value) & Model & Best model \\
\hline $\mathrm{ZIP}$ & P & $7.385(7.65 e-14)$ & $M_{1}>M_{2}$ & $\mathrm{ZIP}$ \\
\hline $\mathrm{ZIP}$ & NB & $4.117(1.92 \mathrm{e}-05)$ & $M_{1}>M_{2}$ & $\mathrm{ZIP}$ \\
\hline ZINB & $P$ & $7.276(1.71 e-13)$ & $M_{1}>M_{2}$ & ZINB \\
\hline ZINB & NB & $4.43(4.69 e-06)$ & $M_{1}>M_{2}$ & ZINB \\
\hline $\begin{array}{l}\text { Pvs. NB } \\
H_{0}: k=0 \text { vs. } H_{1}: k>0\end{array}$ & & & \multicolumn{2}{|c|}{ ZIP VS. ZINB $H_{0}: k=0$ vs. $H_{1}: k>0$} \\
\hline LRT: 203.4174 & & & \multicolumn{2}{|c|}{ LRT: 0.3168} \\
\hline $\begin{array}{l}\text { Best modeling: NB } \\
p=0.000\end{array}$ & & & \multicolumn{2}{|c|}{$\begin{array}{l}\text { Best model: ZIP } \\
p=0.574\end{array}$} \\
\hline
\end{tabular}

significant over dispersion exists; because we would like to divide this value by the degrees of freedom to be close to one.

The Voung test statistic found on Table 3 reflected that all the candidate models, NB, ZIP and ZINB performed better than the standard Poisson model. The ZIP model performed better than NB, better than Poisson and ZINB performed better than NB, better than Poisson. The computed statistic of this test $V=4.12$. Under the null hypothesis this statistic is asymptotically normally distributed. If we consider the significance level of $5 \%$, we conclude easily that ZIP fits the number of ANC visits better than the standard Negative Binomial regression.

For testing over dispersion in Poisson versus NB regressions, $\mathrm{H}_{0}: \mathrm{K}=0$ vs. $\mathrm{H}_{1}: \mathrm{K}>0$, the likelihood ratio statistic $(2[-543.7086-(-645.4173)]=203.42)$ indicating that the null hypothesis is rejected and the NB regression is more adequate since $p$ value $<0.05$. Over dispersion is caused by a positive correlation between ANC or by an excess variation between ANC probabilities or counts. Over-dispersion is also arose when there are violations in the distributional assumptions of the data. For testing over-dispersion in ZIP versus $\mathrm{ZINB}, \mathrm{H}_{0}: \mathrm{K}=0$ vs. $\mathrm{H}_{1}: \mathrm{K}>0$, the likelihood ratio statistic (0.32) indicating that the null hypothesis is accepted and ZIP regressions are more adequate since $\mathrm{p}$-value $>0.05$.

\section{Model selection criteria}

As shown in Table 4 ZIP and ZINB regression models were better fitted than Poisson and NB respectively based on their corresponding AIC. It is found that the models with the smallest AIC and BIC were ZIP regression followed by ZINP regression model since their LR, $\chi^{2}=1017.38$ and $\chi^{2}=1017.70$ were highly significant ( $p$-value $<0.0001$ ) supported by the information criteria.

Figure 2a shows how well the model predicts the count values by overlaying the predicted probabilities for each ANC category on the frequency histogram of the actual ANC data. It appears that the typical regression model under-predicts the 0-10 ANC categories over-predicts all the other categories. The plots of the predicted probability of

Table 4 Model selection criteria for the regression models

\begin{tabular}{lllll}
\hline Criteria & $\mathbf{P}$ & NB & ZIP & ZINB \\
\hline-2 Log & 1290.835 & 1087.417 & 1017.381 & 1017.698 \\
AIC & 1304.835 & 1103.417 & 1045.698 & 1047.381 \\
BIC & 1332.191 & 1134.682 & 1100.411 & 1106.003 \\
\hline
\end{tabular}



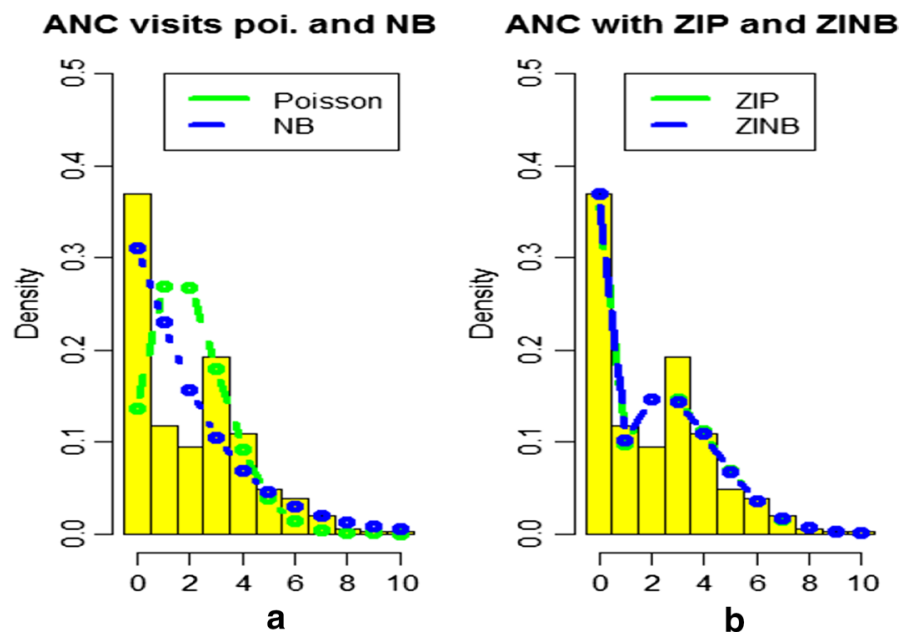

Fig. 2 Comparison of the densities of each model fits. a Number ANC's visits. b Number ANC's visits

each model against the observed probability of the outcome shows that the Poisson and the NB model under-estimated zero counts (Fig. 2a). The zero inflated models captured almost all zero values. Based on predicted probabilities, the differences in model fit between the four models were remarkable. Still the Poisson model and the NB model do not fit the data reasonably well because high zero counts. The Poisson predicted about $27 \%$ zeros and NB model predicted about 33\% zeros compared to ZIP and ZINB about 37\% observed zeros (Fig. 2b).

\section{Parameter estimates and interpretation}

\section{Interpretation (zero-inflated model)}

Table 5, the number of ANC visits become zero for women who had the rural part of pregnant mother been 1.60 times more no ANC visits than the urban pregnant mother. Pregnant mothers who can read and write have lower probability of zero visits of ANC

Table 5 Parameter estimations and SE for the models of ZIP

\begin{tabular}{|c|c|c|}
\hline \multirow[t]{2}{*}{ Parameters } & \multicolumn{2}{|l|}{ Zero-inflated poisson } \\
\hline & $\begin{array}{l}\text { Poisson } \\
\text { Estim. (S.E) }\end{array}$ & $\begin{array}{l}\text { Logit } \\
\text { Estim. (S.E) }\end{array}$ \\
\hline Intercept & $1.2842^{* *}(0.2059540)$ & $0.9996^{* *}(0.6643)$ \\
\hline \multicolumn{3}{|c|}{ Sign of pregnancy (reference-no) } \\
\hline Sign1 & $0.22744^{* *}(0.1634150)$ & $-0.8967(0.5979)$ \\
\hline \multicolumn{3}{|c|}{ Utilization of media (reference-no use of media) } \\
\hline Media1 & $0.1195^{* *}(0.1126641)$ & $-0.5212(0.2495)$ \\
\hline \multicolumn{3}{|c|}{ Mothers education (reference-no education) } \\
\hline M.educ1 & $-0.1297(0.1311842)$ & $0.1314^{* *}(0.3061)$ \\
\hline \multicolumn{3}{|c|}{ Wealth index (reference-poor) } \\
\hline WI2 & $0.00085^{* *}(0.141523)$ & $-0.372^{* *}(0.2914)$ \\
\hline WI3 & $0.28494^{* *}(0.128498)$ & $-0.6181^{* *}(0.293)$ \\
\hline \multicolumn{3}{|c|}{ Area of residence (reference-urban) } \\
\hline Resid2 & $-0.1652^{* *}(0.111293)$ & $0.4702^{* *}(0.276)$ \\
\hline
\end{tabular}


service than those who cannot educate. The estimated odds that the number of ANC visit becomes zero with can read and write is $14.04 \%$ more than the estimated odds for no read and write of pregnant mothers. Wealth index has also statistical relationship with a number of ANC visits. Accordingly, rich women are $46.1 \%$ less odds of having zero ANC visits compared to poor women.

\section{Interpretation (count model)}

Table 5, the expected number of ANC visits of pregnant women from 9 month pregnancy periods for a seen sign of pregnancy complications is 1.2554 times more likely than the expected number of ANC visits for no seen sign of pregnancy complications while holding all other variables in the model constant. The expected number of ANC visits for a use of media is 1.1269 times the expected number of ANC visits for not use of media while holding all other variables in the model constant. That is, the higher utilize media, the fewer predicted ANC visits. The expected number of non zero ANC visits for the rich wealth index would be 1.3297 times the expected number of non-zero ANC visits for the poor wealth index. Similarly the middle wealth index is higher than poor wealth index. The expected number of non zero ANC visits of rural women would be 0.848 times the expected number of non zero ANC visits of the urban women. That means If a subject were to increase rural women by one point, the expected number of ANC visits would decrease by a factor of $\exp (-0.1652)=0.84772$ while holding all other variables in the model constant.

\section{Bayesian zero inflated regression analysis}

A Bayesian approach to investigate the determinate of ANC service visits. For this study, a Gibbs algorithm for all parameters is used. The priors on all parameters are assumed to be Normal $(0, v a r=1000)$. Before using the Bayesian approach results, it is needed to check the convergence assessment that involves checking that the sequence, or chain, has converged to and provides a representative sample from the posterior distribution. We produce a number of graphs which also aid convergence diagnostic checks. The Gibbs sampler algorithm with three simultaneous chains running provide Autocorrelation time series plots of each in different colour help to check convergence. The convergence of the chain can be initially checked visually using trace plots and $\mathrm{MC}$ error in comparison to its posterior standard deviation. The Gelman-Rubin statistic can also be used for checking convergence.

\section{Time series plot}

It is one of the tests used to diagnosis the convergence of Bayesian analysis. Time series plot indicates a good convergence three independent generated channels will mix together or overlapped (Fig. 3 and Appendix: Figs. 6, 7). Here, the diagnostic graphs conclude the simulation draws are reasonably converged and therefore, we can be more confident about the accuracy of posterior inference.

\section{Density plot}

It is another technique for identifying convergence. The plots for all statistically significant co varieties indicated none of the coefficients have bimodal density, and hence the simulated parameter values were converging. 


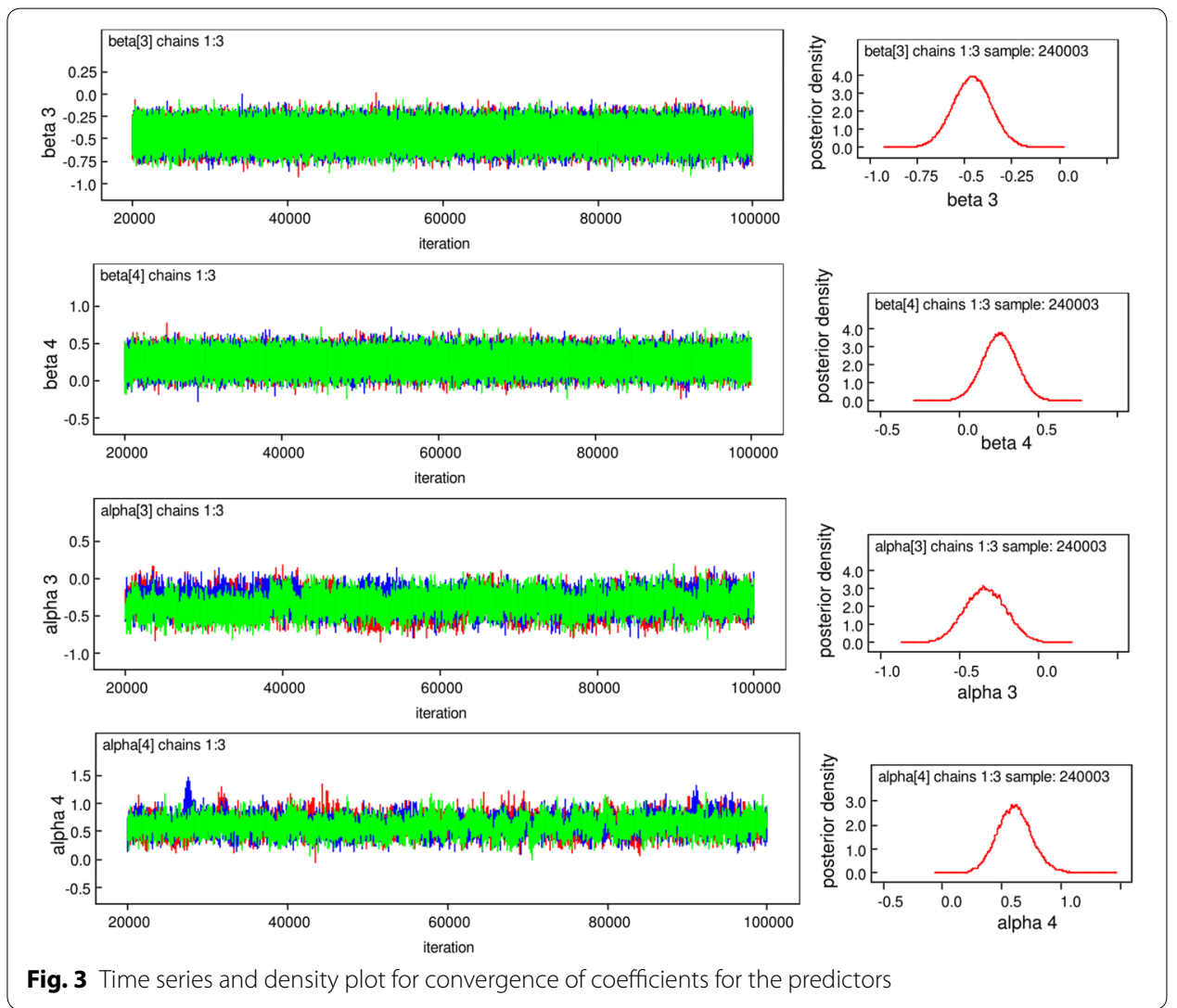

\section{Autocorrelation plot}

It is a test used for convergence of Bayesian analysis. From Fig. 4 and Appendix: Fig. 8, we observe that the autocorrelation for all parameters become law only after considering a lag equal to 50 . Thus, an independent sample can be obtained by rerunning the algorithm with thin set equal to lag 50. Thus, an independent sample can be obtained by rerunning the algorithm with thin set equal to lag 50 . If the 50 lags of three independently generated chains demonstrated, then better convergence indicated. High auto
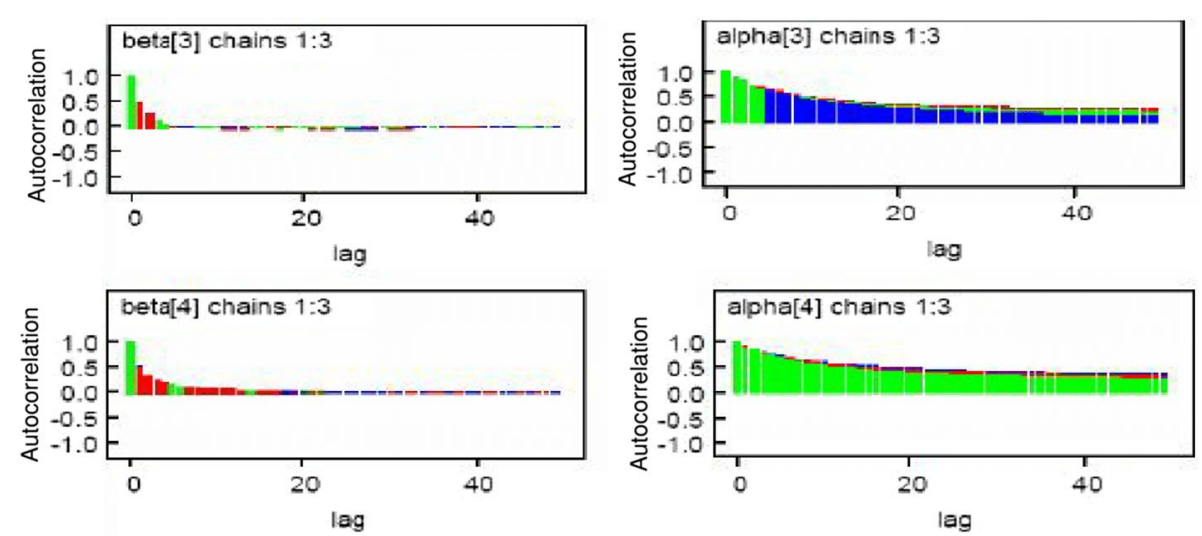

Fig. 4 Auto correlation plot of for convergence of coefficients for the predictors 

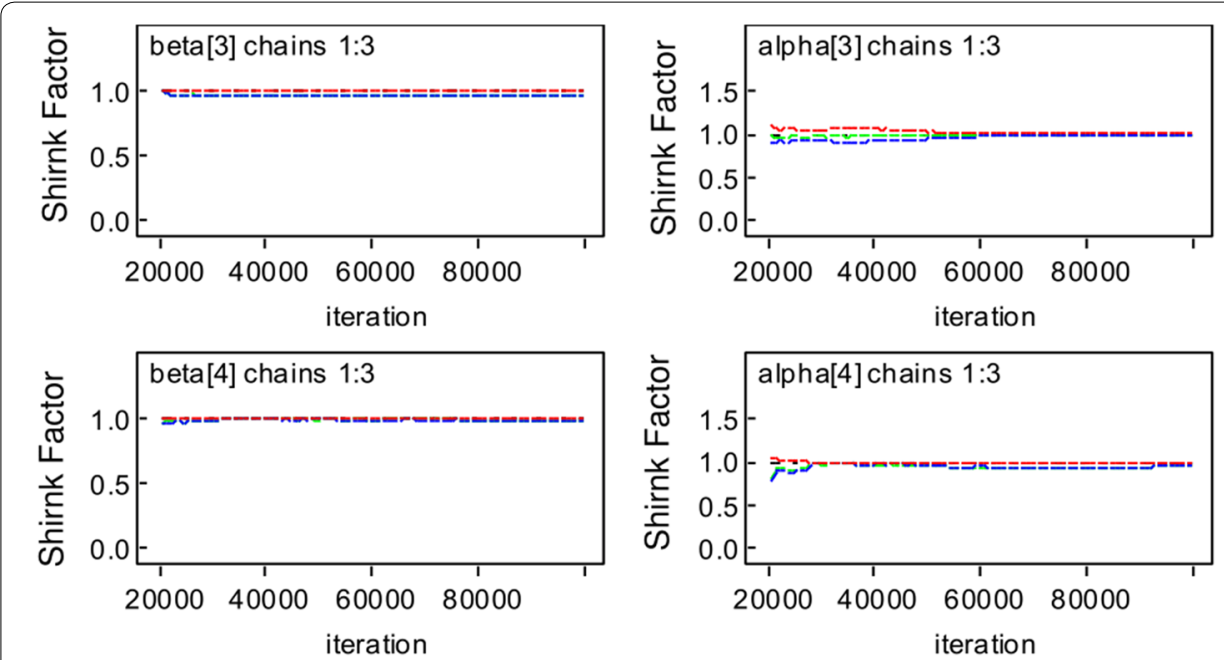

Fig. 5 Gelman-Rubin statistic plot for convergence of coefficients for the predictors

correlations indicate slow mixing within a chain, usually slow convergence to the posterior distribution. The plots show that independent chains were mixed or overlapped to each other.

\section{Gelman-Rubin statistics}

It is one way of checking convergence in Bayesian analysis. It also can be applied only when multiple chains are used. Gelman-Rubin convergence Statistics with the width of the pooled green, the average width of within the individual runs blue and their ratio for plotting purposes the pooled within the interval width are normalized to have an overall maximum of one (Fig. 5). Emphasis that one should be concerned convergence about ratio close to one.

\section{Assessing accuracy of Bayesian ZIP model fitting}

The posterior summary estimates by the MCMC algorithm, especially by Gibbs sampler, like posterior mean, standard errors, Monte carol error, and HPD credible intervals were estimated using winBUGS software. Table 6 contains coefficients of the variables under the column node, the estimated coefficient value under the column mean, the standard error, Monto carol error and HPD credible intervals.

The Gibbs sampler algorithm was implemented with 100,000 iterations in three different chains, 20,000 burn-in terms discarded, as to obtain 240,003 samples from the full posterior distribution. It also provides details concerning the number of iterations revealed as burn-in period and iterations finally kept for estimation. If the MC error value is low in comparison to its posterior standard error, then the posterior density estimates with accuracy. Especially, to have accurate posterior estimates the simulation should be run in until the Monto carol error for each parameter of interest is less than about 5\% of its posterior standard error, and in Bayesian Zero inflated Poisson Regression is accomplished. After, convergence and accuracy of posterior estimates are attained and summarizing the posterior statistics is achievable. 
Table 6 Summary statistic of the posterior distribution of the model parameters

\begin{tabular}{|c|c|c|c|c|c|c|}
\hline \multirow[t]{2}{*}{ Parameters } & \multirow[t]{2}{*}{$\operatorname{Mean}(\hat{\beta})$} & \multirow[t]{2}{*}{ Sd } & \multirow[t]{2}{*}{ MC error } & \multirow[t]{2}{*}{ Median } & \multicolumn{2}{|c|}{ HPD credible intervals } \\
\hline & & & & & Lower (2.5\%) & Upper (97.5\%) \\
\hline \multicolumn{7}{|c|}{ Count model coefficients } \\
\hline$\beta_{1}$ (Intercept) & 1.011 & 0.1795 & 0.001676 & 1.014 & 0.6536 & 1.358 \\
\hline \multicolumn{7}{|c|}{ Sign of pregnancy (reference-no) } \\
\hline$\beta_{2}(\operatorname{Sign} 1)$ & -0.4264 & 0.1555 & 0.001374 & -0.429 & -0.722 & -0.12 \\
\hline \multicolumn{7}{|c|}{ Utilization of media (reference-no use of media) } \\
\hline$\beta_{3}$ (Media1) & -0.4594 & 0.1013 & $3.44 \mathrm{E}-4$ & -0.459 & -0.658 & -0.260 \\
\hline \multicolumn{7}{|c|}{ Mothers' education (reference -no education) } \\
\hline$\beta_{4}($ M.educ1) & 0.257 & 0.1074 & $5.37 \mathrm{E}-4$ & 0.257 & 0.046 & 0.4664 \\
\hline \multicolumn{7}{|c|}{ Wealth index (reference-poor) } \\
\hline$\beta_{5}\left(W I_{2}\right)$ & -0.6421 & 0.1134 & $4.49 \mathrm{E}-4$ & -0.642 & -0.864 & -0.42 \\
\hline$\beta 6\left(\mathrm{Wl}_{3}\right)$ & -0.2698 & 0.1181 & 4.47E-4 & -0.269 & -0.502 & -0.039 \\
\hline \multicolumn{7}{|c|}{ Area of residence (reference-urban) } \\
\hline$\beta_{7}($ Resid2) & 0.3495 & 0.09945 & $3.65 \mathrm{E}-4$ & 0.35 & 0.153 & 0.543 \\
\hline \multicolumn{7}{|c|}{ Zero-inflation coefficients } \\
\hline$a_{1}$ (Intercept) & 0.755 & 0.196 & 0.0042 & 0.7596 & 0.361 & 1.126 \\
\hline \multicolumn{7}{|c|}{ Sign of pregnancy (reference-no) } \\
\hline$a_{2}(\operatorname{Sign} 1)$ & -0.227 & 0.172 & 0.0037 & -0.23 & -0.548 & 0.1259 \\
\hline \multicolumn{7}{|c|}{ Utilization of media (reference-no use of media) } \\
\hline$a_{3}$ (Media1) & -0.335 & 0.130 & 0.0022 & -0.34 & -0.585 & -0.082 \\
\hline \multicolumn{7}{|c|}{ Mothers' education (reference-no education) } \\
\hline$a_{4}$ (M.educ1) & -0.608 & 0.148 & 0.003 & -0.6046 & -0.9153 & -0.330 \\
\hline \multicolumn{7}{|c|}{ Wealth index (reference-poor) } \\
\hline$a_{5}\left(W I_{2}\right)$ & -0.517 & 0.137 & 0.002 & -0.52 & -0.787 & -0.25 \\
\hline$a_{6}\left(W l_{3}\right)$ & 0.04589 & 0.181 & 0.004 & 0.03645 & -0.277 & 0.4431 \\
\hline \multicolumn{7}{|c|}{ Area of residence (reference-urban) } \\
\hline$a_{7}(\operatorname{Resid} 2)$ & 0.124 & 0.1292 & 0.002307 & 0.2392 & 0.116 & 0.3623 \\
\hline
\end{tabular}

In Table $6 \mathrm{MC}$ error for each significant predictor is less than $5 \%$ of its posterior standard error. This implies convergence and accuracy of posterior estimates are attained and the model is appropriate to estimate posterior statistics. In view of the result of with non informative prior given in Table 6, considering the credible interval, the table shows that the following variable; "Utilization of media", "Mothers' education", "Wealth index" and "Residence" are the significant predictors of the determinants of Antenatal Care Service visits Among Pregnant Women. From Bayesian zero inflated Poisson we found out that rural pregnant women are 1.132 times more likely to have no ANC visits compare to urban pregnant women $(\mathrm{OR}=1.132$; HPD CI 1.123, 1.437). Zero inflation interpretation regarding the effects of education on the ANC visits, we found out that can read and write pregnant women are 0.544 times less likely to have no ANC visits compare to no educated pregnant women with the actual significant factors related to ANC visits. Furthermore, as the level of education increases, the odds of having no ANC visit also decrease by 0.544 . Wealth index has also statistical relationship with a number of ANC visits. Accordingly, Middle wealth index women are 59.63\% less odds of having zero ANC visits compared to poor women. The relationship between zero ANC visits and utilization of media exposures are statistically significant. A pregnant Women use of media exposure were 0.7153 times less likely to zero ANC visits than women no use 
of media exposure. The result on Table 6 shows that other factors such as signs of pregnancy are not statistically significant. The result of non informative Bayesian approach shown in Table 6 gives similar significant variables as that of the classical statistics. This is as a result of the presence of previous knowledge in the model.

\section{Model comparison}

Model compares Bayesian ZIP and classical ZIP approaches were identified more significant predictor variables, numerical value different in standard error and other techniques. Before results interpretation terms used in Table 7 like $\mathrm{SE}_{b}$ and $\mathrm{SE}_{\mathrm{C}}$ stands for standard error of Bayesian ZIP and Classical ZIP methods, respectively.

The table shows the comparison of the Bayesian ZIP and Classical ZIP approach of count regression model with their respective error and with a number of significant variables. The predictor variables Utilization of Media, Mothers' education, Wealth index and Residence were statistically significant variables using Bayesian ZIP regression approach and Mothers' education, Wealth index and Residence were statistically significant using classical ZIP regression approach. The important comparison method was using the standard error of both approaches. As, result in the Table 7 given above in the Bayesian ZIP approach of significant variables to the standard error value is less than the

Table 7 The model comparison based on standard error

\begin{tabular}{|c|c|c|c|c|c|}
\hline \multirow[t]{2}{*}{ Variable (coefficients) } & \multicolumn{2}{|l|}{ BZIP } & \multicolumn{2}{|l|}{ CZIP } & \multirow[t]{2}{*}{ SE comparison } \\
\hline & Mean $(\hat{\beta})$ & $\mathrm{SE}_{\beta}$ & $\hat{\beta}$ & SEC & \\
\hline \multicolumn{6}{|l|}{ Count model coefficients } \\
\hline$\beta_{1}$ (Intercept) & 1.011 & 0.00936 & 1.2842217 & 0.2059540 & $\mathrm{SE}_{\mathrm{B}}<\mathrm{SE}_{\mathrm{C}}$ \\
\hline \multicolumn{6}{|c|}{ Sign of pregnancy (reference-no) } \\
\hline$\beta_{2}(\operatorname{Sign} 1)$ & -0.4264 & 0.008106 & -0.2274489 & 0.1634150 & $\mathrm{SE}_{\mathrm{B}}<\mathrm{SE}_{\mathrm{C}}$ \\
\hline \multicolumn{6}{|c|}{ Utilization of media (reference-no use of media) } \\
\hline$\beta_{3}$ (Media1) & -0.4594 & 0.00528 & -0.1195001 & 0.1126641 & $\mathrm{SE}_{\mathrm{B}}<\mathrm{SE}_{\mathrm{C}}$ \\
\hline \multicolumn{6}{|c|}{ Mothers' education (reference-no education) } \\
\hline$\beta_{4}($ M.educ1)) & 0.257 & 0.005599 & 0.1297838 & 0.1311842 & $\mathrm{SE}_{\mathrm{B}}<\mathrm{SE}_{\mathrm{C}}$ \\
\hline \multicolumn{6}{|c|}{ Wealth index (reference-poor) } \\
\hline$\beta_{5}(\mathrm{~W} \mid 2)$ & -0.6421 & 0.0059115 & -0.0008455 & 0.1415231 & $\mathrm{SE}_{\mathrm{B}}<\mathrm{SE}_{\mathrm{C}}$ \\
\hline$\beta_{6}(\mathrm{~W} \mid 3)$ & -0.2698 & 0.0061565 & -0.2849420 & 0.1284985 & $\mathrm{SE}_{\mathrm{B}}<\mathrm{SE}_{\mathrm{C}}$ \\
\hline \multicolumn{6}{|c|}{ Area of residence (reference-urban) } \\
\hline$\beta_{7}($ Resid2) & 0.3495 & 0.005184 & -0.1651500 & 0.1112927 & $\mathrm{SE}_{\mathrm{B}}<\mathrm{SE}_{\mathrm{C}}$ \\
\hline \multicolumn{6}{|l|}{ Zero-inflation coefficients } \\
\hline$a_{1}$ (Intercept) & 0.755 & 0.01022 & -0.9996 & 0.6643 & $\mathrm{SE}_{\mathrm{B}}<\mathrm{SE}_{\mathrm{C}}$ \\
\hline \multicolumn{6}{|c|}{ Sign of pregnancy (reference-no) } \\
\hline$a_{2}(\operatorname{Sign} 1)$ & -0.227 & 0.008966 & 0.8967 & 0.5979 & $\mathrm{SE}_{\mathrm{B}}<\mathrm{SE}_{\mathrm{C}}$ \\
\hline \multicolumn{6}{|c|}{ Utilization of media (reference-no use of Media) } \\
\hline$a_{3}$ (M.educ1) & -0.335 & 0.0067768 & 0.5212 & 0.2495 & $\mathrm{SE}_{\mathrm{B}}<\mathrm{SE}_{\mathrm{C}}$ \\
\hline \multicolumn{6}{|c|}{ Mothers' education (reference-no education) } \\
\hline$a_{4}($ M.educ1) & -0.608 & 0.0077152 & -0.1314 & 0.3061 & $\mathrm{SE}_{\mathrm{B}}<\mathrm{SE}_{\mathrm{C}}$ \\
\hline \multicolumn{6}{|c|}{ Wealth index (reference-poor) } \\
\hline$a_{5}\left(W I_{2}\right)$ & -0.517 & 0.0071417 & -0.3718 & 0.2914 & $\mathrm{SE}_{\mathrm{B}}<\mathrm{SE}_{\mathrm{C}}$ \\
\hline$a_{6}\left(W I_{3}\right)$ & 0.04589 & 0.009435 & -0.6181 & 0.2932 & $\mathrm{SE}_{\mathrm{B}}<\mathrm{SE}_{\mathrm{C}}$ \\
\hline \multicolumn{6}{|c|}{ Area of residence (reference-urban) } \\
\hline$a_{7}(\operatorname{Resid} 2)$ & 0.124 & 0.0067351 & 0.4702 & 0.2758 & $\mathrm{SE}_{\mathrm{B}}<\mathrm{SE}_{\mathrm{C}}$ \\
\hline
\end{tabular}


classical ZIP approach. Based on the standard error value Bayesian ZIP approach is a better than classical ZIP method.

\section{Discussion}

In health service studies, Antenatal care service visits could be a relevant metric to quantify efficiency of maternal care utilization. This paper, which focused on an efficient statistical modeling for number of Antenatal care service visits, Bayesian zero-inflated poison modeling approach to estimate parameters of demographic, socio-economic, health and environmental related factors. This study has focused on assessing the status of ANC utilization and modeling Bayesian count regression model of the determinants of utilization of antenatal care service visits among pregnant women in Amhara regional state. The study showed that $63 \%$ of the pregnant women have been received at least one ANC during their periods of pregnancy. As compared to the 2011 data, there is a $22 \%$ increase in the percentage of women who have been received ANC [10]. This increment may be attributed to awareness creation, health promotion, and increase in the number of health institutions and health care providers in the rural areas. Studies conducted in Dembecha district and Debre Tabor town, Northern Ethiopia showed that 57-55.7\% of pregnant women have been received at least one ANC respectively. This little difference may be due to the time difference and strength of data collection. As the World Health Organization recommended, in the absence of complications, every woman should have at least four ANC visits [33]. However, fewer than one-third (27\%) of pregnant women had four or more visits during the length of their pregnancy. According to the 2011 EDHS report, only 19\% of women were received four or more ANC visits [10]. Although this finding shows a low level of service utilization, there is remarkable improvement as compared to the 2011 national rate. This discrepancy may be due to fact that awareness of respondents is increased, an increase in the promotion of health, and increase in the number of health institutions and health care providers in the region. The time difference between the current study and the previous studies can be one attributable factor. The ZIP regression model has been developed and utilized to handle data of this type, and is traditionally estimated by using maximum likelihood. This paper introduces a Bayesian alternative to estimate the ZIP model, as we believe it provides several advantages when compared to maximum likelihood estimation for this model. For example, as interval estimates receive increasing emphasis in health science research, the commonsense interpretation of Bayesian intervals (i.e., credible intervals) provides a strong impetus to adopt a Bayesian perspective [25]. Perhaps more importantly in the case of the ZIP model, Bayesian analysis can provide the full joint distribution of the parameters of interest and can account for various sources of uncertainty in modeling zero-inflated count data, which is not easily achieved in classical maximum likelihood approaches [25]. Small samples along with parameters close to the boundary may result in extra uncertainty in parameters and failure of the asymptotic assumptions, which are critical in maximum likelihood estimation. These conditions may cast doubt on statistical inferences about model parameters for count data with excess zero values based on the variance of the estimator under maximum likelihood approaches [25]. Our simulation studies demonstrate that the Bayesian method performs better in the sense of yielding larger coverage probabilities and smaller bias than the classic maximum likelihood 
approach, especially in the case of small samples with either very high or very low incidence of excess zero outcomes. From the intervals and the table as well, it is evident that there is a noticeable amount of zero inflation in residence, mother's education, middle wealth index and media exposure because the interval of concentration of the posterior distributions do not include zero, where as for signs of pregnancy the posterior distribution of $\mathrm{p}$ is centered around zero and the interval contains zero, signifying the absence of zero inflation (Table 6). Finally, for the sake of completeness, Table 6 gives 0.95 credible intervals and HPD intervals calculated from the posteriors. It is seen that for the media, mothers' education and middle wealth index and residence that 0 is not in the intervals. This is consistent with the presence of zero inflation. For the Sign of pregnancy, 0 is in the interval. The interval is so wide much of it includes negative values. So, it is not surprising that zeroes inflation is not indicated by the test. In this study the use of antenatal care was found to be related to economic status; Mothers with middle economic level were more likely to attend ANC than poor women. This is also in line with other studies conducted in Southern Ethiopia [2]. In this study, it was found that Bayesian zero inflated poison regression were better fitted the data than NB and Poisson.

\section{Conclusion}

Bayesian inference and frequented (classical) statistics findings are difficult to compare, the reason that they are different techniques with different tools for decision making, standard deviation and confidence interval for one and credible interval for the other. However, findings from the Bayesian model is given preference because the technique is more robust and precise than the traditional statistics. In conclusion, the Bayesian ZIP model was better fitted the data which can be considered a mixture of two statistical processes, one always generating zero ANC visits and the other generating both variability in the zero and non-zero ANC visits than any other models and therefore it was selected as the best parsimonious model. The ANC service utilization rate in the rural Amhara region is lower than the national figures available to date. In addition, it is worth nothing that majority of the mothers who attend ANC did not receive an adequate number of visits recommended by the World Health Organization. Furthermore, Using Bayesian ZIP analysis mother's education, media exposure, residence and the wealth index were major predictors of ANC service utilization.

\footnotetext{
Abbreviations

WHO: World Health Organization; ANC: antenatal care services; OR: odds ratio; MCMC: Markov chain Monte Carlo; ZIP: zero-inflated Poisson; MDGs: millennium development goals; EMDHS: Ethiopia Mini Demographic and Health Survey; EDHS: Ethiopia Demographic and Health Survey; winBUGS: win Bayesian using Gibbs sampling; Cl: confidence interval; HPD: highest posterior density; CSA: Central Statistical Agencies; NB: negative binomial; ZINB: zero inflated negative binomial.

Authors' contributions

MS made substantial contributions to the conception and design of the study, formulation the methodology, analyzed and interpreted the data, and was a major contributor in writing the manuscript. AM designed the study, collected the data, supervised and reviewed the final version submitted for publication. Both authors read and approved the final manuscript.

Author details

${ }^{1}$ Mathematical and Statistical Modeling, Debre Markos University, Debre Markos, Ethiopia. ${ }^{2}$ Public Health in Epidemiology and Biostatistics, Debre Markos University, Debre Markos, Ethiopia.
} 
Acknowledgements

The authors wish to acknowledge Debre Markos University for funding this study. Our deepest gratitude goes to Central statistical Agency for providing the data.

\section{Competing interests}

The authors declare that they have no competing interests.

\section{Availability of data and materials}

The datasets used and/or analyzed during the current study available from the corresponding author on reasonable request.

\section{Consent for publication}

Authors prove consent of publication for this research.

Ethics approval and consent to participate

Not applicable.

Funding

This work was supported by Debre Markos University and No payment is due on publication of this article. The article processing charge has been waived by Springer Open).

\section{Appendix}

See Table 8, Figs. 6, 7, 8.

Table 8 Parameter estimations and S.E for the models of P, NB, ZIP, ZINB

\begin{tabular}{|c|c|c|c|c|c|c|}
\hline \multirow[t]{3}{*}{ Parameters } & \multirow[t]{2}{*}{$\mathbf{P}$} & \multirow[t]{2}{*}{ NB } & \multicolumn{2}{|l|}{ ZIP } & \multicolumn{2}{|l|}{ ZINB } \\
\hline & & & \multirow{2}{*}{$\begin{array}{l}\text { Poisson } \\
\text { Estim. (S.E) }\end{array}$} & \multirow{2}{*}{$\begin{array}{l}\text { Logit } \\
\text { Estim. (S.E) }\end{array}$} & \multirow{2}{*}{$\begin{array}{l}\text { NegBinomal } \\
\text { Estim. (S.E) }\end{array}$} & \multirow{2}{*}{$\begin{array}{l}\text { Logit } \\
\text { Estim. } \\
\text { (S.E) }\end{array}$} \\
\hline & Estim. (S.E) & Estim. (S.E) & & & & \\
\hline Intercept & $\begin{array}{r}0.76058^{* * *} \\
(0.18592)\end{array}$ & $\begin{array}{l}1.0383^{* *} \\
(0.4298)\end{array}$ & $\begin{array}{l}1.2842^{* *} \\
(0.2059540)\end{array}$ & $\begin{array}{c}-0.9996^{* *} \\
(0.6643)\end{array}$ & $\begin{array}{l}1.27952^{* *} \\
(0.2174236)\end{array}$ & $\begin{aligned}- & 1.0171^{* *} \\
& (0.6739)\end{aligned}$ \\
\hline \multicolumn{7}{|c|}{ Sign of pregnancy (reference-no) } \\
\hline Sign1 & $\begin{array}{r}-0.4377^{* *} \\
(0.15539)\end{array}$ & $\begin{array}{c}-0.6216^{* *} \\
(0.3823)\end{array}$ & $\begin{array}{l}0.22744^{* *} \\
(0.1634150)\end{array}$ & $\begin{array}{r}-0.8967 \\
(0.5979)\end{array}$ & $\begin{array}{c}-0.22983^{* *} \\
(0.1723128)\end{array}$ & $\begin{array}{l}0.8985^{* *} \\
(0.6052)\end{array}$ \\
\hline \multicolumn{7}{|c|}{ Utilization of media (reference-no use of media) } \\
\hline Media1 & $\begin{array}{c}-0.3702^{* * *} \\
(0.10100)\end{array}$ & $\begin{array}{r}-0.405^{* *} \\
(0.1860)\end{array}$ & $\begin{array}{l}0.1195^{* *} \\
(0.1126641)\end{array}$ & $\begin{array}{r}-0.5212 \\
(0.2495)\end{array}$ & $\begin{array}{r}-0.121061^{* *} \\
(0.1172531)\end{array}$ & $\begin{array}{l}0.5219^{* *} \\
(0.2518)\end{array}$ \\
\hline \multicolumn{7}{|c|}{ Mothers' education (reference-no education) } \\
\hline M.educ1 & $\begin{array}{r}0.27931^{* * *} \\
(0.10757)\end{array}$ & $\begin{array}{l}0.1077^{* *} \\
(0.2229)\end{array}$ & $\begin{array}{l}-0.1297 \\
(0.1311842)\end{array}$ & $\begin{array}{l}0.1314^{* *} \\
(0.3061)\end{array}$ & $\begin{array}{l}0.131155^{*} \\
(0.1376872)\end{array}$ & $\begin{array}{r}-0.1288 \\
(0.3096\end{array}$ \\
\hline \multicolumn{7}{|c|}{ Wealth index (reference-poor) } \\
\hline$W l_{2}$ & $\begin{array}{l}0.2419^{*} \\
(0.12278)\end{array}$ & $\begin{array}{r}0.1775^{* * *} \\
(0.2171)\end{array}$ & $\begin{array}{l}0.00085^{* *} \\
(0.1415231)\end{array}$ & $\begin{array}{c}-0.3718^{* *} \\
(0.2914)\end{array}$ & $\begin{array}{c}0.0009002^{* *} \\
(0.1467337)\end{array}$ & $\begin{array}{c}-0.3731^{* *} \\
(0.2943)\end{array}$ \\
\hline $\mathrm{Wl}_{3}$ & $\begin{array}{l}0.5902^{* * *} \\
(0.11172)\end{array}$ & $\begin{array}{l}0.6099^{* *} \\
(0.2151)\end{array}$ & $\begin{array}{l}0.28494^{* *} \\
(0.1284985)\end{array}$ & $\begin{array}{c}-0.6181^{* *} \\
(0.2932)\end{array}$ & $\begin{array}{r}0.2865452^{* *} \\
(0.134157)\end{array}$ & $\begin{array}{r}-0.6174^{* *} \\
(0.2961)\end{array}$ \\
\hline \multicolumn{7}{|c|}{ Area of residence (reference-urban) } \\
\hline Resid2 & $\begin{array}{r}-0.368^{* * *} \\
(0.09945)\end{array}$ & $\begin{array}{c}-0.4145^{* *} \\
(0.2005)\end{array}$ & $\begin{array}{l}-0.1652^{* *} \\
(0.1112927)\end{array}$ & $\begin{array}{l}0.4702^{* *} \\
(0.2758)\end{array}$ & $\begin{array}{r}-0.166245^{* *} \\
(0.1163618)\end{array}$ & $\begin{array}{l}0.4707^{* *} \\
(0.2787)\end{array}$ \\
\hline $\begin{array}{l}\text { Log-likeli- } \\
\text { hood }\end{array}$ & -645.4173 & -543.7086 & -508.8491 & & -508.6907 & \\
\hline Dispersion & 2.6666 & 0.9009 & 1.1159 & & 1.0991 & \\
\hline
\end{tabular}

${ }^{*} p<0.05,{ }^{* *} p<0.01,{ }^{* * *} p<0.001$ 

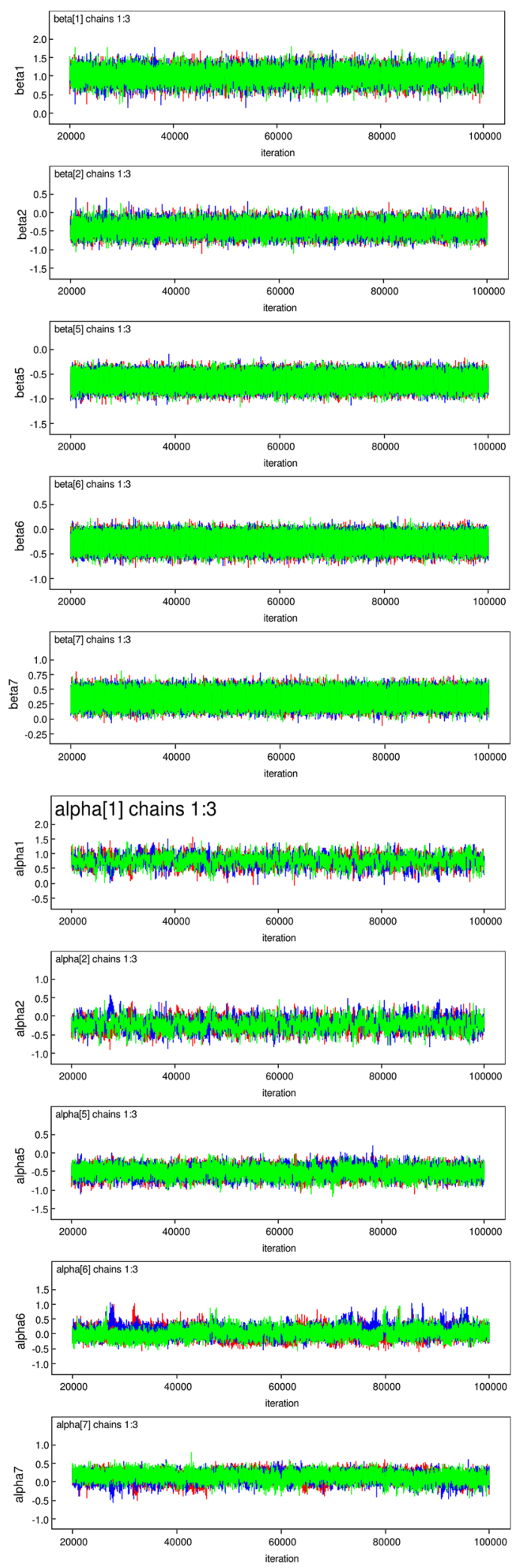

Fig. 6 Time series for convergence of coefficients for the predictors 






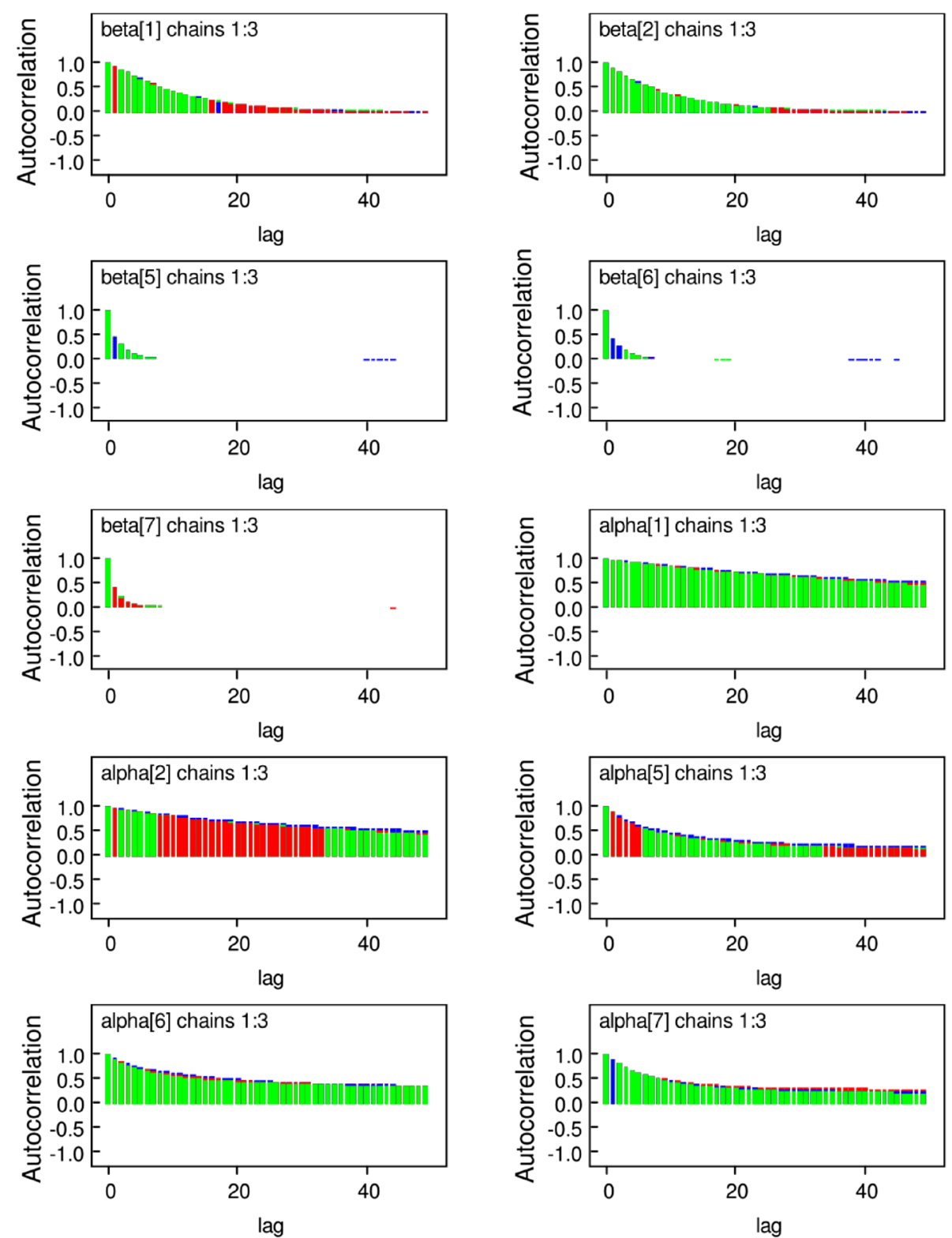

Fig. 8 Auto correlation plot for convergence of coefficients for the predictors

\section{Publisher's Note}

Springer Nature remains neutral with regard to jurisdictional claims in published maps and institutional affiliations.

Received: 4 December 2017 Accepted: 31 January 2018

Published online: 24 February 2018

\section{References}

1. Bloom SS, Lippeveld T, Wypij D. Does antenatal care make a difference to safe delivery? A study in urban Uttar Pradesh, India. Health Policy Plan. 1999;14(1):38-48.

2. Abosse Z, Woldie M, Ololo S. Factors influencing antenatal care service utilization in hadiya zone. Ethiop J Health Sci. 2010;20(2):75-82. 
3. Raatikainen K, Heiskanen N, Heinonen S. Under-attending free antenatal care is associated with adverse pregnancy outcomes. BMC Public Health. 2007;7:268.

4. Lawn J, Kerber K. Opportunities for Africas newborns: practical data policy and programmatic support for newborn care in Africa. 2006.

5. Trinh LTT, Rubin G. Late entry to antenatal care in New South Wales, Australia. Reprod Health. 2006;3(1):8.

6. Who U. UNFPA (2004) Maternal mortality in 2000: estimates developed by WHO, UNICEF, and UNFPA. Geneva: World Health Organization; 2003.

7. Organization WH, United Nations Children's Fund. Accountability for maternal, newborn and child survival: The 2013 Update. Countdown to 2015 maternal, newborn and child survival. Geneva: WHO Press; 2013. p. 2013.

8. Dalal K, Shabnam J, Andrews-Chavez J, Mårtensson LB, Timpka T. Economic empowerment of women and utilization of maternal delivery care in Bangladesh. Int J Prev Med. 2012:3(9):628.

9. Who U. World bank: trends in maternal mortality: 1990-2010. Geneva: WHO, UNICEF. UNFPA and The World Bank estimates; 2012

10. Demographic E. Health Survey: Addis Ababa. Ethiopia and Calverton, Maryland, USA: central statistics agency and ORC macro. 2011.

11. Fatema N. Importance of antenatal care. MO, CMH, Chittagong, Bangladish). According to Ethiopian Demographic and Health Survey (EDHS) 2011. 2010.

12. Mo Health. AIDS in Ethiopia, sixth report. Ethiopia: Addis-Ababa; 2006.

13. Assefa E, Tadesse M. Factors related to the use of antenatal care services in Ethiopia: application of the zero-inflated negative binomial model. Women Health. 2017;57(7):804-21.

14. Edhs M. Ethiopia Mini Demographic and Health Survey. Ethiopia: Central Statistical Agency Addis Ababa; 2014.

15. Bayou YT, Mashalla YS, Thupayagale-Tshweneagae $G$. The adequacy of antenatal care services among slum residents in Addis Ababa, Ethiopia. BMC Pregnancy Childbirth. 2016;16(1):142.

16. Rurangirwa AA, Mogren I, Nyirazinyoye L, Ntaganira J, Krantz G. Determinants of poor utilization of antenatal care services among recently delivered women in Rwanda; a population based study. BMC Pregnancy Childbirth. 2017;17(1):142.

17. Ethiopia FDRoo. Ministry of Health Ministry of Health. 2007.

18. Hilbe JM. Modeling count data. International encyclopedia of statistical science. Berlin: Springer; 2011. p. 836-9.

19. Ridout M, Demétrio CG, Hinde J, editors. Models for count data with many zeros. Proceedings of the XIXth international biometric conference; 1998.

20. Washington SP, Karlaftis MG, Mannering F. Statistical and econometric methods for transportation data analysis. Boca Raton: CRC Press; 2010.

21. Lambert D. Zero-inflated Poisson regression, with an application to defects in manufacturing. Technometrics. 1992;34(1):1-14

22. Ismail N, Jemain AA. Handling overdispersion with negative binomial and generalized Poisson regression models. Casualty actuarial society forum. Citeseer: Princeton; 2007.

23. Vuong QH. Likelihood ratio tests for model selection and non-nested hypotheses. Econom J Econom Soc. 1989;57(2):307-33.

24. Congdon P. Bayesian models for categorical data. Hoboken: Wiley; 2005.

25. Gelman A, Carlin J, Stern H, Rubin D. Bayesian data analysis. Chapman and Hall/CRC Press; 2004

26. Rodrigues J. Bayesian analysis of zero-inflated distributions. Commun Stat Theory Methods. 2003;32(2):281-9.

27. Ghosh SK, Mukhopadhyay P, Lu J-CJ. Bayesian analysis of zero-inflated regression models. J Stat Plan Inference. 2006;136(4):1360-75.

28. Dagne GA. Hierarchical Bayesian analysis of correlated zero-inflated count data. Biom J. 2004:46(6):653-63.

29. Gilks WR, Richardson S, Spiegelhalter D. Markov chain Monte Carlo in practice. Boca Raton: CRC Press; 1995.

30. Spiegelhalter D, Thomas A, Best N, Gilks W. BUGS 0.5: Bayesian inference using Gibbs sampling manual (version ii). Cambridge: MRC Biostatistics Unit Institute of Public Health; 1996. p. 1-59.

31. Albert J. Bayesian computation with R. Berlin: Springer Science \& Business Media; 2009.

32. Walsh B. Introduction to Bayesian analysis. Lecture notes for EEB 596z. 2002.

33. Organization WH. Health worker role in providing safe abortion care and post abortion contraception. Geneva: World Health Organization; 2015.

\section{Submit your manuscript to a SpringerOpen ${ }^{\circ}$ journal and benefit from:}

- Convenient online submission

- Rigorous peer review

- Open access: articles freely available online

High visibility within the field

- Retaining the copyright to your article

Submit your next manuscript at $\boldsymbol{\nabla}$ springeropen.com 